\title{
Temperature and gravity of the pulsating extreme helium star LSS 3184 (BX Cir) through its pulsation cycle ${ }^{\star}$
}

\author{
V. M. Woolf ${ }^{\star \star}$ and C. S. Jeffery \\ Armagh Observatory, College Hill, Armagh, BT61 9DG, Northern Ireland \\ e-mail: vmw@astro.washington.edu,csj@star.arm.ac.uk \\ Received 6 May 2002 / Accepted 30 July 2002

\begin{abstract}
We report the analysis of optical spectra of the extreme helium star LSS 3184 (BX Cir) to determine its effective temperature and gravity throughout its pulsation cycle. The spectra were also used to measure its chemical abundances. using an optical spectrum with lower spectral resolution and a lower signal to noise ratio. Our analysis decreases the upper limit for the $\mathrm{H}$ abundance to $\mathrm{H}<6.0$ (mass fraction $<7.1 \times 10^{-7}$ ). Our gravity corresponds to stellar mass $M=0.47 \pm 0.03 M_{\odot}$. We find that the effective $\log g$ varies through the pulsation cycle with an amplitude of 0.28 dex. The effective gravity is smaller than the rest gravity except when the star is very near its minimum radius. The change in effective gravity is primarily caused by acceleration of the stellar surface.

Based on the optical spectra, we find the temperature varies with an amplitude of $3450 \mathrm{~K}$. We find a time averaged mean temperature, $23390 \pm 90 \mathrm{~K}$, consistent with that found in the earlier optical spectrum study. The mean temperature is $1750 \mathrm{~K}$ hotter than that found using combined ultraviolet spectra and $V$ and $R$ photometry and the variation amplitude is larger. This discrepancy is similar to that found for the extreme helium star V652 Her.
\end{abstract} \\ We report rest gravity, $\log g=3.38 \pm 0.02$, and a chemical abundance mixture consistent with those reported earlier in a study
}

Key words. stars: chemically peculiar - stars: oscillations - stars: variables - stars: individual: LSS 3184 - stars: atmospheres

\section{Introduction}

Extreme Helium stars (EHes) are a class of low-gravity, hot, evolved stars with very large helium abundances ( $~ 299$ per cent) and weak or non-existent hydrogen lines. They appear to be rapidly evolving to become white dwarfs. The short time the stars spend as EHes explains why few EHes are known, despite their brightness $\left(L \gtrsim 900 L_{\odot}\right)$.

The evolutionary history of EHes is still uncertain. The more popular proposals have been that they are formed by the merger of two white dwarfs (Webbink 1984; Iben \& Tutukov 1984) or that they are the result of a "late thermal pulse" when the helium shell near the surface of a white dwarf ignites, causing the star to expand (Iben et al. 1983). Saio \& Jeffery (2002) have recently shown that the white dwarf merger model is the most likely explanation. Determining the history of EHes will provide clues to the evolutionary history of possibly related stars (RCrB stars, He-rich subdwarfs, carbon stars, etc.). As the chemical abundance mixture in EHes appears to be the end result of a combination of $\mathrm{CNO}$ and triple- $\alpha$ processing, their

Send offprint requests to: V. M. Woolf,

e-mail: vmw@star.arm.ac.uk

^ Based on observations obtained at the Anglo-Australian Telescope, Coonabarabran, NSW, Australia.

$\star \star$ Current address: Astronomy Department, University of Washington, Box 351580, Seattle, WA 98195-1580, USA study may provide information about physical processes occurring in a large fraction of stars.

Some EHes pulsate. The pulsations are driven through the $\kappa$ mechanism, with iron group (Z-bump) elements providing the needed opacity (Saio 1995). Pulsation provides additional methods to study the physical properties of stars. Radial velocities, ultraviolet spectra, and optical photometry have been used to determine the radius, gravity, temperature, mass, and absolute magnitude of two pulsating EHes: LSS 3184 and V652 Her (Lynas-Gray et al. 1984; Kilkenny et al. 1999; Woolf \& Jeffery 2000; Jeffery et al. 2001). Mass and chemical composition are the parameters which best constrain evolutionary models for EHes. In previous studies of LSS 3184 the relatively large uncertainty for the gravity produced most of the uncertainty for the mass determination.

In a recent study of pulsation models for EHes, Montañéz Rodríguez \& Jeffery (2002) showed that the pulsation period and radial velocity curve of LSS 3184 require a mass between 0.38 and $0.5 M_{\odot}$ if the temperature is assumed to be between 22400 and $24000 \mathrm{~K}$. However, the models with those parameters also require a luminosity smaller than half of that previously measured.

In this paper we report the determination of chemical abundances and time resolved temperature and effective gravity of LSS 3184 using spectral analysis of the optical spectra used to find radial velocities in Woolf \& Jeffery (2000). 


\section{Observations and data reduction}

The optical spectra used in this study are those used to determine radial velocities through the pulsation cycle of LSS 3184 as described in Woolf \& Jeffery (2000). The spectra were obtained at the Anglo-Australian Telescope on the nights of 1996 May 18 and 19 using the University College London Echelle Spectrograph. Standard IRAF packages were used for bias and flat field correction, reducing echelle orders to one dimensional spectra, and applying the wavelength scale using thorium-argon arc spectra. The spectral resolution is $\lambda / \Delta \lambda \approx$ 48000 .

We merged the echelle orders to produce a single spectrum for each observation covering the entire observed wavelength range. This is a fairly complicated procedure because the fluxes rarely match and frequently have opposite slopes in the sections of the echelle orders which overlap in wavelength. This is caused mostly by incomplete correction for the grating blaze function.

Combining the echelle orders without properly correcting this produces discontinuities at the wavelengths at which echelle orders begin and end. Normalizing the echelle orders by dividing by estimates of the continuum levels before merging reduces the size of the discontinuities but does not eliminate them. Furthermore, because there are uncertainties in continuum fitting, using this method makes it difficult to determine if a difference between two spectra is caused by stellar variability or by an inconsistency in continuum fitting. This problem is worse when a strong absorption feature appears near or at the end of an echelle order.

To overcome this problem, we coadded the spectra to produce an average spectrum with a high signal to noise ratio. Continuum shapes were estimated for each echelle order where it was reasonable to do so, i.e. orders without strong absorption features. Second degree polynomials, $y=a+b x+c x^{2}$, were sufficient to reproduce the curvatures present. Fifth degree polynomials were then fitted to the coefficient $(a, b, c)$ vs. echelle order curves and used to calculate "smoothed" continuum fit coefficients for all orders, including those with strong absorption features.

The smoothed continuum fits calculated with the average spectrum were then used to normalize individual spectra. The IRAF procedure SCOMBINE was used to combine the echelle orders into a single normalized spectrum without the problem of discontinuities previously present.

This problem has been treated by others using somewhat different methods (e.g. Barker 1984; Erspamer \& North 2002).

\section{Analysis}

We determined that the individual spectra were too noisy to be used to find the temperature, gravity, and chemical composition of LSS 3184 reliably. We combined the spectra into twelve phase bins to provide spectra with high enough signal to noise ratios $(S T N)$ for our analysis $(30<S T N<50)$ while also providing small enough time increments to allow us to measure changes to the atmospheric conditions through the pulsation cycle. The bins were selected so that the second one is centered at minimum radius (phase $=0.1)$. Bins are one twelfth of a cycle long $(P=0.1065784 \mathrm{~d})$ (Kilkenny et al. 1999) and contain between 10 and 15 individual spectra.

Determining microturbulent velocity was an iterative process. $\mathrm{C}$ and $\mathrm{O}$ abundances were found using absorption line equivalent widths and estimates of $\log g$ and $T_{\text {eff }}$ in the program SPECTRUM (Jeffery et al. 2001). Microturbulence was varied until there was no slope in the $\mathrm{C}$ and $\mathrm{O}$ abundance vs equivalent width plots. We calculated $\log g$ and $T_{\text {eff }}$ assuming this microturbulence (Sect. 3.1). We then tested that the previously determined microturbulence produced $\mathrm{C}$ and $\mathrm{O}$ abundance vs. equivalent width plots with no slope with the newly determined $\log g$ and $T_{\text {eff. }}$

We found $v \sin i$ by fitting line widths in synthetic spectra to those in the optical spectra. Other line-broadening effects which do not affect line saturation such as macroturbulence and pulsation velocity change during the exposure may be included in the number we found. Based on the accelerations measured in Woolf \& Jeffery (2000), the surface velocity change during a 5 min exposure is about $1 \mathrm{~km} \mathrm{~s}^{-1}$ for most of the pulsation cycle and about $12 \mathrm{~km} \mathrm{~s}^{-1}$ near minimum radius. Including this velocity drift in our analysis had no substantial appreciable effect on the temperatures or gravities we calculated.

\subsection{Temperature and gravity}

We determined the temperature and effective gravity of LSS 3184 for each phase bin using the FORTRAN90 program SFIT (Jeffery et al. 2001). In sFIT interpolated synthetic spectra are fitted to observed spectra to find the best $T_{\text {eff }}$ and $\log g$ values. The input spectra were calculated for a fixed chemical composition and microturbulent velocity $\xi=6.9 \mathrm{~km} \mathrm{~s}^{-1}$.

Finding the best $T_{\text {eff }}$ and $\log g$ values for a phase bin was a two step process. In both steps we used $v \sin i=15.8 \mathrm{~km} \mathrm{~s}^{-1}$. In the first step we let $T_{\text {eff }}$ vary while holding $\log g$ fixed and used the wavelength regions without strong helium lines. We found the $T_{\text {eff }}$ values which produced the best fits for various input $\log g$ values. In the second step we set SFIT to let $\log g$ vary while holding $T_{\text {eff }}$ fixed and to use the wavelength regions with strong helium lines. We then found the $\log g$ values which produced the best fits for various input $T_{\text {eff }}$ values. Examples of spectral fits for He lines and metal lines are shown in Fig. 1. (We note that modeling the cores of strong $\mathrm{He}$ lines in hot $\mathrm{He}$ stars is a continuing problem.) We estimated the temperature and gravity for each phase bin by plotting the $T_{\text {eff }}$ vs. $\log g$ data for the two steps and finding the intersection of the two sets (Fig. 2). Uncertainty in the spectral data and the analysis caused intersections to occur over a range of $T_{\text {eff }}$ and $\log g$. We can therefore only determine that the best estimate lies within the intersection range. We believe that a large part of this uncertainty occurs because the spectral fitting routine adjustments to the continuum level of the observed spectrum can improperly cancel the effects of $\log g$ differences.

The $\log g$ values we measured are effective $\log g$ values: $\log g_{\mathrm{eff}}=\log \left(g_{\text {rest }}+a\right)$ where $\log g_{\text {rest }}$ is the gravitational acceleration, $G M r^{-2}$, which would be measured for the star at a current radius if it were not pulsating, and $a$ is the $\mathrm{d}^{2} r / \mathrm{d} t^{2}$ 


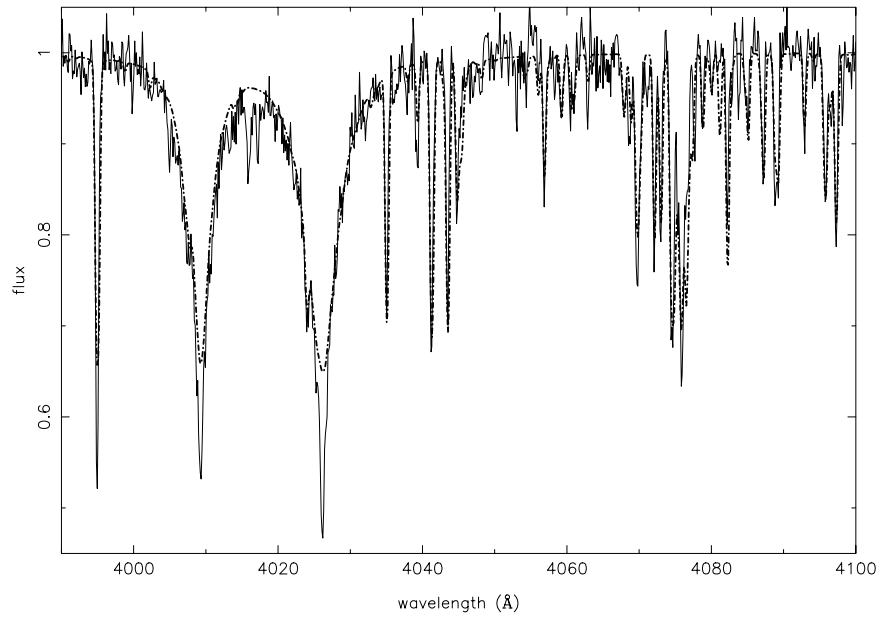

Fig. 1. Observed (solid line) and synthetic (dotted line) spectra of He lines at 4009 and $4026 \AA$ and some metal lines. The observed spectrum is for phase 0.6 (bin 8). The synthetic spectrum was calculated for $T=21750, \log g=3.08$.

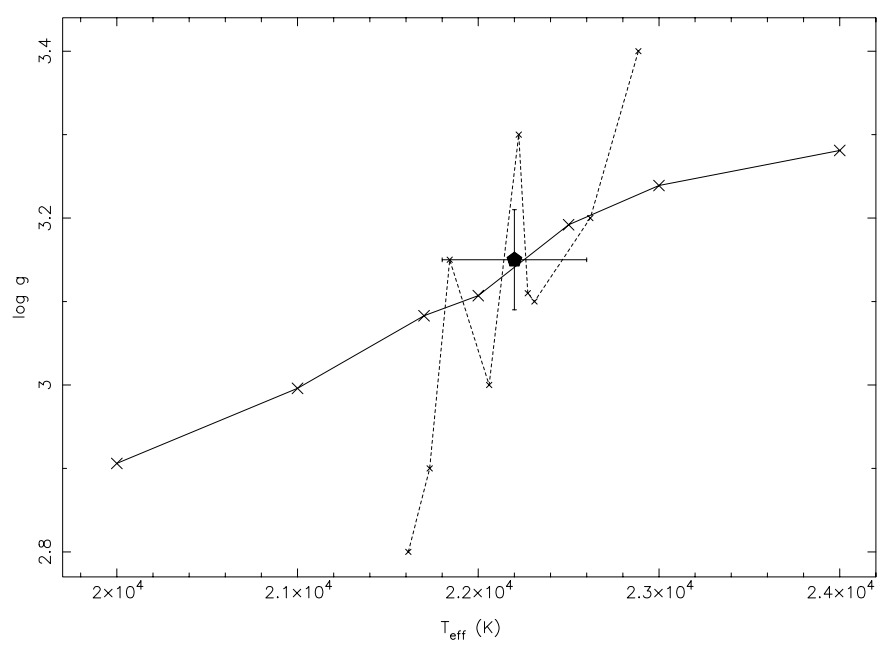

Fig. 2. $T_{\text {eff }}$ found using fixed $\log g$ (dotted line) and $\log g$ found using fixed $T_{\text {eff }}$ (solid line) for bin 10 (phase: $0.775<\phi<0.80833$ ). The large data point shows the adopted $T_{\text {eff }}$ and $\log g$ values and uncertainties for the bin values.

acceleration of the surface of the pulsating star. (To avoid confusion, hereafter we will refer to $\log g_{\text {rest }}$ as "gravity" and $\mathrm{d}^{2} r / \mathrm{d} t^{2}$ as "acceleration".) The gravity for each phase bin can be determined by subtracting the acceleration from the measured $\log g_{\text {eff }}$. Figure 3 displays $\log g_{\text {eff }}, \mathrm{d}^{2} r / \mathrm{d} t^{2}$, and $\log g_{\text {rest }}$ through the pulsation cycle. The phase bin at maximum acceleration (minimum radius) does not have a data point in the upper panel because the radial acceleration is larger than the effective gravity measured, giving a negative gravity. The points in the upper panel with $X$ 's were not used to calculate the average $\log g_{\text {rest }}$ : they appear to be affected by the atmosphere being out of equilibrium as a result of the strong compression caused by radial acceleration near minimum radius. The error bars indicate formal measurement errors. They do not include systematic errors caused by uncertainties in the assumptions used or incomplete physics in our analysis. As will be discussed in the results section, there is obviously some important physics omitted in our analysis of the star near minimum radius.

\subsection{Chemical composition}

The chemical composition of LSS 3184 should not change with pulsation phase. We calculated elemental abundances using the combined spectra from the four phase bins closest to maximum radius (minimum temperature) when the atmosphere is changing more slowly and where assuming local thermodynamic equilibrium conditions is more reasonable. The spectral wavelengths were corrected to zero velocity prior to co-adding. This provided a representative spectrum with a high signal to noise ratio $(S T N \sim 100)$ for abundance analysis.

A model atmosphere was calculated for the mean temperature and gravity found previously for the four bins. We calculated atomic abundances using this model atmosphere and the FORTRAN90 program SFIT_SYNTH (Jeffery et al. 2001) to fit synthetic spectra to the combined observed spectrum. Fe was an exception. With only three Fe III lines (4139.4, 4164.8, and $4419.6 \AA$ ) unblended and strong enough for analysis we used the program SPECTRUM to find the $\mathrm{Fe}$ abundance based on their equivalent widths. We did not find any $\mathrm{Mg}$ lines of adequate quality to determine the $\mathrm{Mg}$ abundance.

\section{Results: physical properties of LSS 3184}

The effective temperature and effective gravity for each phase bin are listed in Table 1. The uncertainties quoted for our data are formal errors based on how precisely the models predict temperature and gravity. They do not include uncertainties in the physics that went into the models. Near minimum radius where non-equilibrium effects occur the model uncertainties are large.

The variation of effective gravity with phase is displayed in Fig. 3. The difference in radius at maximum and minimum provides a gravity change of only 0.04 dex in $\log g$, so the effective gravity variation is primarily caused by acceleration of the star's surface due to pulsation. When we use spectroscopy to measure gravity, what we are actually measuring is the pressure in the atmosphere as manifested in the wings of strong lines (He lines in EHes). Acceleration of the atmosphere produces corresponding pressure changes which are small when the acceleration is small, so that the assumption of hydrostatic equilibrium in the model atmosphere is reasonable. But at minimum radius and maximum acceleration the measured gravity does not increase as much as expected and we calculate a negative gravity. This is probably because one of the assumptions used in determining the gravity, local equilibrium, is clearly not valid when the atmosphere is undergoing a strong, fast acceleration and compression. We believe that the gravity values found at minimum radius and nearby phase bins (missing or marked with $X$ 's in the top panel of Fig. 3) are inaccurate because of these effects.

The temperature variation is compared to the variation found by fitting UV and visible flux levels (Woolf \& Jeffery 2000 ) in Fig. 4. The temperatures found using spectral analysis of visual spectra are hotter by more than $1600 \mathrm{~K}$ than 

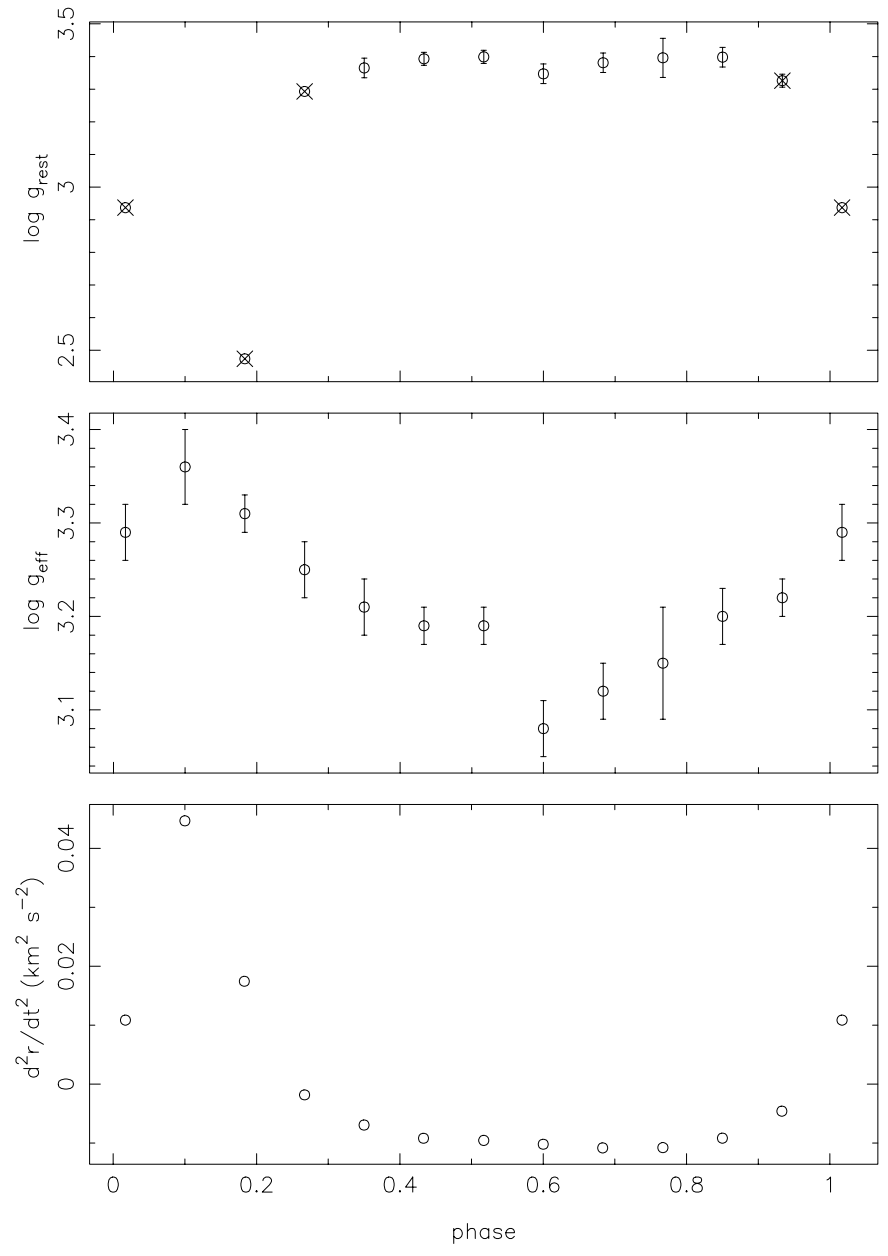

Fig. 3. Linear surface acceleration (bottom panel), effective gravity (middle panel), and gravity (top panel) of LSS 3184 through its pulsation cycle. The $\log g_{\text {rest }}$ panel does not have a point at phase $=0$ because radial acceleration there is larger than the measured effective gravity, giving a negative gravity.

those found using visual and UV fluxes for both LSS 3184 and V652 Her. The temperature variation amplitude based on visible spectra is also larger. This problem is discussed for V652 Her in Jeffery et al. (2001).

The temperature curve found by Kilkenny et al. (1999) using optical photometry is very similar to the one we find using optical spectral analysis. Montañéz Rodríguez \& Jeffery (2002) found that models which fitted the velocity and flux curves of LSS 3184 well required a mass between 0.38 and $0.50 M_{\odot}$ and a temperature between 22300 and $23900 \mathrm{~K}$. Because analysis of optical spectra, optical photometry, and theoretical models each predict the hotter temperature, it appears that the lower temperature estimate found using UV and visual fluxes is probably in error. That a nearly identical discrepancy was found for V652 Her means that this is probably a systematic problem. We suspect that incomplete line opacity in our models is the main cause.

The physical parameters and chemical abundances derived for LSS 3184 are presented in Table 2 and are compared with those found in previous studies and with those of the Sun, "typical" hot EHes, and V652 Her. V652 Her is a hot EHe
Table 1. Temperature and gravity through the pulsation cycle of LSS 3184. Phases are specified at center of phase bins.

\begin{tabular}{lllll}
\hline \hline bin & phase & $T_{\text {eff }}(\mathrm{K})$ & $\log g_{\text {eff }}$ & $\log g_{\text {rest }}$ \\
\hline 1 & 0.0167 & $24600 \pm 250$ & $3.29 \pm 0.03$ & 2.94 \\
2 & 0.1000 & $25200 \pm 500$ & $3.36 \pm 0.04$ & \\
3 & 0.1833 & $24690 \pm 300$ & $3.31 \pm 0.02$ & 2.47 \\
4 & 0.2667 & $24250 \pm 300$ & $3.25 \pm 0.03$ & 3.29 \\
5 & 0.2500 & $23450 \pm 300$ & $3.21 \pm 0.03$ & 3.36 \\
6 & 0.4333 & $22750 \pm 200$ & $3.19 \pm 0.02$ & 3.39 \\
7 & 0.5167 & $22400 \pm 100$ & $3.19 \pm 0.02$ & 3.40 \\
8 & 0.6000 & $21750 \pm 350$ & $3.08 \pm 0.03$ & 3.35 \\
9 & 0.6833 & $21900 \pm 300$ & $3.12 \pm 0.03$ & 3.38 \\
10 & 0.7667 & $22200 \pm 400$ & $3.15 \pm 0.06$ & 3.40 \\
11 & 0.8500 & $23050 \pm 350$ & $3.20 \pm 0.03$ & 3.40 \\
12 & 0.9333 & $24400 \pm 200$ & $3.22 \pm 0.02$ & 3.33 \\
\hline
\end{tabular}

with pulsation period, temperature, and radius nearly identical to those of LSS 3184. The errors quoted for our data are formal measurement errors and do not account for systematic errors due to uncertainties in our input assumptions or incomplete physics in our calculations. The parameters we report are consistent with those found previously using optical spectra with higher noise, lower spectral resolution, and no time resolution. We found a larger gravity, though it is within the uncertainty quoted for the previous determination. The new gravity combined with the stellar radius found in Woolf \& Jeffery (2000) produces a mass estimate 12 per cent larger than that found using the previously determined $\log g$.

The elemental abundances found for LSS 3184, V652 Her, and typical hot EHes are shown in Fig. 5. The Mg abundance for LSS 3184 is taken from Drilling et al. (1998). In the figure we can see that LSS 3184 has a much lower $\mathrm{H}$ abundance and V652 Her has a much lower C abundance than the others. The $\mathrm{H}$ abundance we report for LSS 3184 is an upper limit: we could find no evidence for any $\mathrm{H}$ absorption lines in our spectra. As an example, the observed spectrum is compared to synthetic spectra with different $\mathrm{H}$ abundances in the spectral region including the Balmer $\mathrm{H} \beta$ line in Fig. 6 . The weak feature at $4860.97 \AA$ is an O II line and is fitted well with no H contribution. The $\mathrm{H}$ line strengths become negligible for $\mathrm{H}<6.0$ (mass fraction less than one part in $1.4 \times 10^{6}$ ).

It is also evident from Fig. 5 that LSS 3184 has a lower metallicity than V652 Her and typical hot EHes. It has a lower abundance of all metals measured except that V652 Her has a smaller $\mathrm{C}$ abundance, probably as the result of $\mathrm{CNO}$ processing its $\mathrm{C}$ to $\mathrm{N}$, and the typical EHe Fe abundance is smaller. We believe the metallicity differences indicate differences in metallicity of the progenitor stars.

Saio \& Jeffery $(2000,2002)$ showed that the white dwarf (WD) merger model can explain the chemical abundances observed in EHes. V652 Her shows evidence of the CNO process converting essentially all carbon and oxygen into nitrogen. LSS 3184 and typical hot EHes show evidence of CNO processing with high $\mathrm{N}$ abundances, but also show evidence of the triple- $\alpha$ process adding new C. Saio \& Jeffery say this this could be because V652 Her is the result of a He-He WD merger 
Table 2. Physical parameters of LSS 3184 compared with previous determinations and with V652 Her, Sun and average hot extreme helium stars. Abundances use normalization: $\log \sum_{i} \mu_{i} n_{i}=12.15$. Uncertainties listed are formal errors and do not take into account uncertainties of input parameters or model assumptions.

\begin{tabular}{|c|c|c|c|c|c|c|}
\hline & LSS 3184 & LSS $3184(\mathrm{WJ})$ & LSS $3184(\mathrm{KD})$ & V652 Her & hot EHe & Sun \\
\hline Period (days) & & & 0.1065784 & 0.10799319 & & \\
\hline$\left\langle T_{\mathrm{eff}}\right\rangle(\mathrm{K})$ & $23390 \pm 90$ & 21640 & $23300 \pm 700$ & $22930^{c}$ & $>13000$ & \\
\hline$\langle\log g\rangle$ & $3.38 \pm 0.02$ & & $3.35 \pm 0.10$ & 3.7 & & \\
\hline$\langle R\rangle\left(R_{\odot}\right)$ & & $2.31 \pm 0.10$ & $1.35 \pm 0.15$ & $2.31 \pm 0.02$ & & \\
\hline $\operatorname{mass}\left(M_{\odot}\right)$ & $0.47 \pm 0.03$ & $0.42 \pm 0.12$ & 0.12 to 0.42 & $0.59 \pm 0.19$ & & \\
\hline$\langle L\rangle\left(L_{\odot}\right)$ & $1432 \pm 88$ & $1400 \pm 300$ & $485 \pm 135$ & $919 \pm 14$ & & \\
\hline $\mathrm{H}$ & $<6.0$ & & $<7.72$ & 9.5 & 8.0 & 12.00 \\
\hline $\mathrm{He}$ & 11.54 & & 11.54 & 11.54 & 11.54 & 10.99 \\
\hline $\mathrm{C}$ & $8.89 \pm 0.16$ & & 9.02 & 7.03 & 9.3 & 8.58 \\
\hline $\mathrm{N}$ & $8.43 \pm 0.13$ & & 8.26 & 8.9 & 8.3 & 8.05 \\
\hline $\mathrm{O}$ & $7.87 \pm 0.10$ & & 8.05 & 7.9 & 8.6 & 8.93 \\
\hline $\mathrm{Mg}$ & & & 7.17 & 8.1 & 7.6 & 7.58 \\
\hline $\mathrm{Al}$ & $5.91 \pm 0.07$ & & 6.04 & 6.7 & 6.1 & 6.47 \\
\hline $\mathrm{Si}$ & $6.59 \pm 0.11$ & & 6.91 & 7.7 & 7.4 & 7.55 \\
\hline $\mathrm{P}$ & $4.86 \pm 0.08$ & & 4.96 & 5.8 & 5.7 & 5.45 \\
\hline S & $6.59 \pm 0.11$ & & 6.67 & 7.4 & 7.1 & 7.33 \\
\hline $\mathrm{Fe}$ & $6.72 \pm 0.13$ & & 6.52 & 7.4 & 5.8 & 7.50 \\
\hline
\end{tabular}

Table references:

WJ LSS 3184 data: Woolf \& Jeffery (2000) (UV and visible flux).

KD LSS 3184 data: Kilkenny et al. (1999), Drilling et al. (1998).

V652 Her: Lynas-Gray et al. (1984), Jeffery et al. (1986), Jeffery (1996), Jeffery et al. (2001).

Sun: Grevesse et al. (1996).

average hot EHe: Pandey et al. (2001).

${ }^{c}$ or $20950 \mathrm{~K}$ using UV and visible flux.

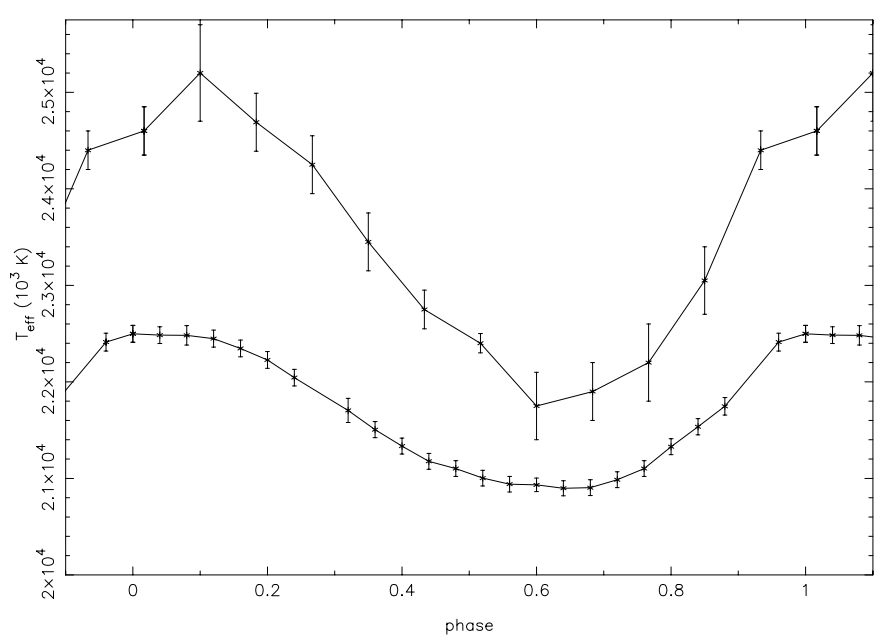

Fig. 4. $T_{\text {eff }}$ vs. phase for LSS 3184 . The upper curve displays the results from this paper. The lower curve displays results of UV and visible flux fitting in Woolf \& Jeffery (2000).

while LSS 3184 and typical hot EHes are the result of He-CO WD mergers. A He-He WD merger for V652 Her would also help explain other differences (e.g. the luminosity) between it and other EHes, including LSS 3184.

Although the gravity we find for for LSS 3184 is larger than that previously determined, the mass to which it corresponds, $M=0.47 \pm 0.03 M_{\odot}$, is still smaller than that permitted by

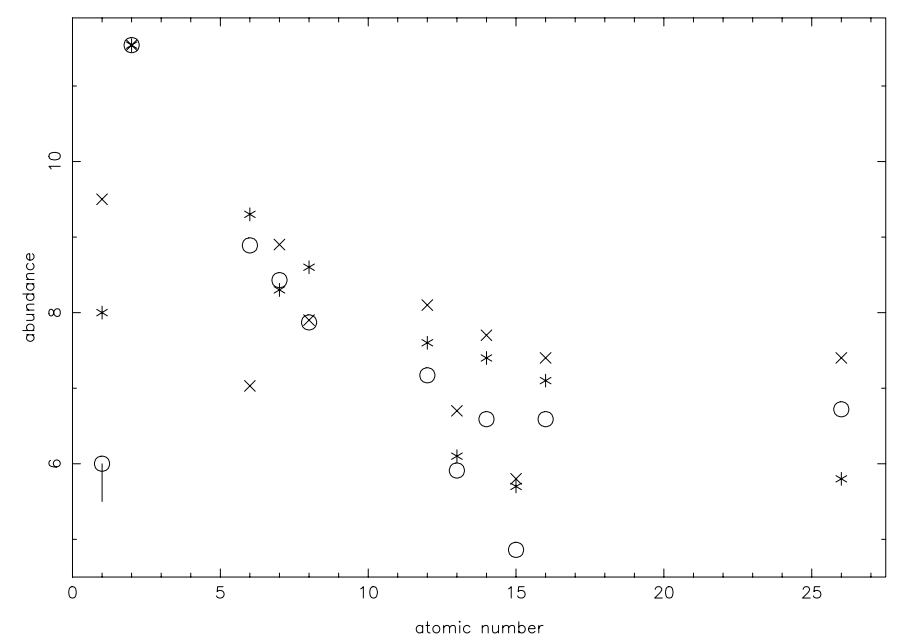

Fig. 5. Chemical abundances of LSS 3184 (circles), V652 Her (crosses), and typical hot EHes (asterisks) vs. atomic number. The $\mathrm{H}$ abundance for LSS 3184 is an upper limit.

WD merger models for EHe production. It is unclear whether improved merger models or gravities found with an improved model atmosphere program will remove this discrepancy or if this will rule out the WD merger model.

The hydrodynamic models of LSS 3184 which best match its pulsation period and radial velocity curve for temperatures between 22400 and 24000 require a mass between 0.38 


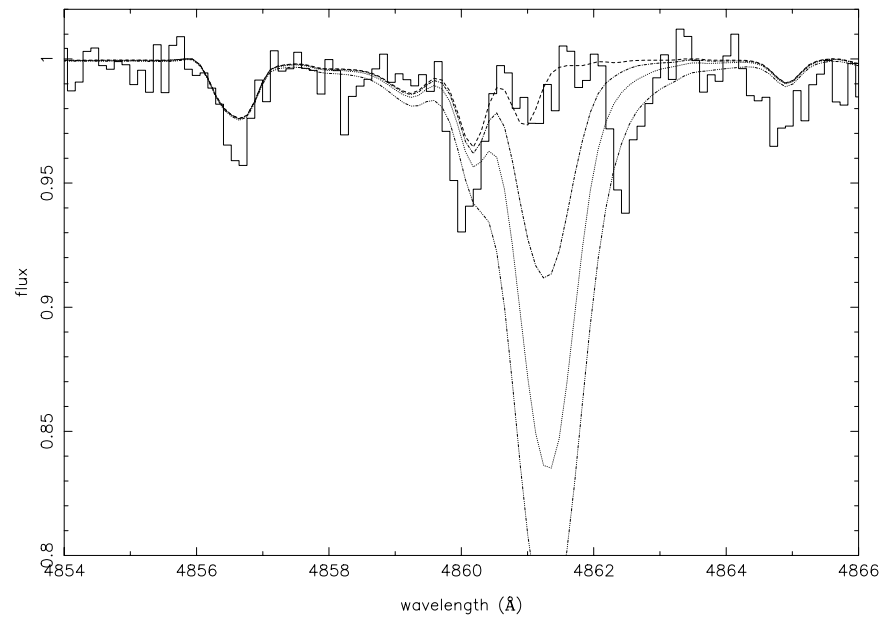

Fig. 6. $\mathrm{H}-\beta$ region of observed LSS 3184 spectrum and synthetic spectra with $\mathrm{H}=4.0,6.0,8.0,8.5$, and 9.0. $\mathrm{H}=4.0$ and 6.0 are identical.

and $0.5 M_{\odot}$ (Montañéz Rodríguez \& Jeffery 2002). The mass we find here using optical spectral analysis $\left(0.47 \pm 0.03 M_{\odot}\right)$ and the mass we found by fitting synthetic spectral fluxes to UV spectra and optical photometry $\left(0.42 \pm 0.12 M_{\odot}\right)$ Woolf \& Jeffery (2000) are comfortably within this range.

However, the Montañéz Rodríguez \& Jeffery models also predict a luminosity $2.74<\log L / L_{\odot}<2.84$ smaller than half of the luminosity found using the temperature and radius from Woolf \& Jeffery (2000), $\log L / L_{\odot}=3.15 \pm 0.09$. We do not derive a new luminosity in this paper. Kilkenny et al. (1999) found $2.54<\log L / L_{\odot}<2.79$ using optical photometry. Most of the difference between the luminosities are caused by the differences in stellar radius used in the calculations: $1.519<$ $R / R_{\odot}<1.708$ from the pulsation models, $R=1.35 \pm 0.15 R_{\odot}$ from optical photometry, and $R=2.35 \pm 0.10 R_{\odot}$ from UV plus optical flux fitting.

We suspect that the match between the model luminosity and the optical photometry luminosity and the mismatch with the luminosity based on UV plus optical flux fitting is another indication that there is a problem with the part of our code that deals with UV flux.

\section{Conclusions}

We have used time resolved spectra with high signal to noise ratios and high spectral resolution to determine the temperature, gravity, and chemical composition of LSS 3184. Our results are consistent with those of previous analyses based on optical spectra and photometry. We have reduced the uncertainties on some of these quantities.
We have reduced the upper limit on the hydrogen abundance by a factor of 52 to $\mathrm{H}<6.0$. Our gravity and mass estimates are 12 per cent larger than previous estimates.

The time averaged mean temperature estimate we obtain with our spectral analysis is $1750 \mathrm{~K}$ hotter than that obtained by fitting UV and visible flux levels. A similar temperature discrepancy was found for V652 Her. The problem is probably the result of incomplete line opacity in our model stellar atmospheres.

The mass and temperature we find for LSS 3184 is consistent with those expected based on the pulsation models of Montañéz Rodríguez \& Jeffery (2002) and with those found using optical photometry. The discrepancy between the luminosity expected from the models and that calculated based on UV observations remains a problem.

The chemical composition of LSS 3184 corresponds well to that expected from a white dwarf merger. However, its mass is smaller than the merger models currently allow.

Acknowledgements. We acknowledge financial support from the Northern Ireland Department of Culture, Arts, and Leisure and the UK PPARC (grant Ref PPA/G/S/1998/00019).

\section{References}

Barker, P. K. 1984, ApJ, 89, 899

Drilling, J. S., Jeffery, C. S., \& Heber, U. 1998, A\&A, 329, 1019

Erspamer, E., \& North, P. 2002, A\&A, 383, 227

Grevesse, N., Noels, A., \& Sauval, A. J. 1996, in Cosmic Abundances, ed. S. S. Holt, \& G. Sonneborn, ASP Conf. Ser., 99, 117

Iben, I. Jr., Kaler, J. B., Truran, J. W., \& Renzini, A. 1983, ApJ, 264, 605

Iben, I. Jr., \& Tutukov, A. V. 1984, ApJS, 55, 335

Jeffery, C. S. 1996, in Hydrogen-deficient Stars, ed. C. S. Jeffery, \& U. Heber, ASP Conf. Ser., 96, 152

Jeffery, C. S., Heber, U., \& Hill, P. W. 1986, in IAU Coll. 87, Hydrogen-deficient Stars and Related Objects, ed. K. Hunger, D. Schönberner, \& N. K. Rao (Dordrecht, Holland), 101

Jeffery, C. S., Woolf, V. M., \& Pollacco, D. L. 2001, A\&A, 376, 497

Kilkenny, D., Koen, C., Jeffery, C. S., Hill, N. C., \& O’Donoghue, D. O. 1999, MNRAS, 310, 1119

Lynas-Gray, A. E., Schönberner, D., Hill, P. W., \& Heber, U. 1984, MNRAS, 209, 387

Montañéz Rodríguez, P., \& Jeffery, C. S. 2002, A\&A, 384, 433

Pandey, G., Rao, N. K., Lambert, D. L., Jeffery, C. S., \& Asplund, M. 2001, MNRAS, 324, 937

Saio, H. 1995, MNRAS, 277, 1393

Saio, H., \& Jeffery, C. S. 2000, MNRAS, 313, 671

Saio, H., \& Jeffery, C. S. 2002, MNRAS, 333, 121

Webbink, R. F. 1984, ApJ, 277, 355

Woolf, V. M., \& Jeffery, C. S. 2000, A\&A, 358, 1001 